\title{
Mechanical and Dynamic Mechanical Properties of Sheets Made from Micro-Fibrillated Cellulose and Cationic Polyacrylamide
}

\author{
Takushi Sakaemura ${ }^{* 1}$ and Tatsuo Yamauchi ${ }^{* 2, \#}$ \\ ${ }^{* 1}$ R\&D Department, Arakawa Chemical Industries, LTD., 1-1-9, Tsurumi, Tsurumi-ku, Osaka 538-0053, Japan \\ ${ }^{* 2}$ Graduate School of Agriculture, Kyoto University, Sakyo-ku, Kyoto 606-8502, Japan
}

\begin{abstract}
A diluted micro-fibrillated cellulose (MFC) dispersion with cationic polyacrylamide (C-PAM) aq. solution was casted and dried as a plausible model material of fiber-to-fiber bonds in paper containing C-PAM. Mechanical and dynamic mechanical properties of the MFC/C-PAM mixture sheets (MFC/C-PAM sheets) obtained were studied, comparing with those of papers containing C-PAM. The high density and Young's modulus of the MFC/C-PAM sheet, which was twice those of the paper without C-PAM, suggested higher consolidation and fully developed fibril-fibril bonds due to the quite small dimensions of MFC. A significant decrease in scattering coefficient of the MFC/C-PAM sheets accompanied by an increase of C-PAM content suggested that almost all of the C-PAM in the MFC/C-PAM sheets could locate surrounding fibril-fibril bonds. Tensile strength of MFC/C-PAM sheet was far larger than that of the paper without C-PAM. However, the strength increment of the MFC/C-PAM sheets with increasing C-PAM content was smaller than that expected from the difference of Young's modulus between MFC/C-PAM sheets and the papers containing C-PAM, partly because C-PAM itself is brittle. The dynamic mechanical properties of the MFC/C-PAM sheets were, on the whole, similar to those of the paper containing C-PAM, suggesting that the role of C-PAM on the mechanical property (or bonding) was minimal and the most of it was derived from fibril-fibril (hydrogen) bonding in the MFC/C-PAM sheets. Measurement of (dynamic) mechanical properties of the sheets consisted of MFC and various water soluble polymers could be used to evaluate the effects of the polymers on mechanical properties of papers when the polymers were used as dry strength agents.
\end{abstract}

(Received 30 September, 2013 ; Accepted 25 December, 2013)

\section{Introduction}

In the last decade the manufacturing and application of so-called cellulose nano-fiber (CNF) or microfibrillated cellulose (MFC) have been extensively investigated $^{1,2}$. MFC was initially prepared through mechanical pressure-drop homogenization ${ }^{3)}$, and then chemical pre-treatment methods, such as TEMPO oxidation, were developed to reduce the required energy $\operatorname{cost}^{4}$. Although various applications of MFC were proposed, composite material with polymer has been a leading commercial application. However, a poor distribution (agglomeration) of MFC has been an unavoidable problem in its application, due to a lack of affinity between cellulose and polymers. Among numerous commercially available polymers, polyacrylamide polymer (PAM) has been used for a long time as a dry strength resin in paper materials. This means that PAM possesses good affinity with cellulose and thus

\# corresponding author a mixture sheet of MFC and PAM was recently developed $^{5}$. In the previous studies on cationic PAM (CPAM) as a paper additive ${ }^{6-8)}$, the effects of addition of CPAM and its distribution within a fiber wall or paper sheet on strength properties of papers were investigated in order to understand the mechanism of dry strength development of paper containing C-PAM. The results suggested that C-PAM played a role to enhance bond strength per unit bonded area between fibers as well as increasing the bonded area in some extent. Both led to an increase in paper strength $^{6}$. Furthermore, the C-PAM distributed on and/or nearby the surface of fibers particularly affected the development of paper strength ${ }^{7,8)}$. Pulp fiber used in papermaking is constituted of cellulose micro fibrils as a bundle of CNF or MFC, and thus C$\mathrm{PAM}$ is adsorbed onto micro and nano fibrils extending from wet pulp fibers when C-PAM is added into the pulp slurry. After drying, a somewhat homogenous mixture of C-PAM and nano fibrils could be formed nearby fiber-tofiber bonds. The mixture may affect mechanical properties of the fiber-to-fiber bonds and further strength 
properties of the resulting paper. Conversely, properties of the nano fibril/C-PAM mixed sheets could be expected to reflect the properties of fiber-to-fiber bonds in paper containing C-PAM ${ }^{9)}$.

In this study, sheets were made from mixtures of a commercially available MFC and a cationic C-PAM as a sort of model of fiber-to-fiber bonds in paper rather than a model sheet of a homogenous mixture of polymer and MFC. And its mechanical and dynamic mechanical properties were investigated, comparing with those of paper containing C-PAM.

\section{Experimental}

\subsection{Materials}

A commercially available MFC (CELISH KY-100G, Daicel Chemical Industries, Ltd., Japan) was used without further purification. The C-PAM used in this study was referred to a previous report ${ }^{7)}$. MFC was diluted to $1 \%$ concentration on mass with de-ionized water as well as $\mathrm{C}$ PAM. MFC dispersion was continuously stirred for $2 \mathrm{hrs}$ before mixing with C-PAM. MFC and C-PAM were mixed at given ratios (MFC/C-PAM $=100 / 0,99 / 1$, 98/2, $97 / 3,95 / 5$ on mass as solid). Thereafter, the mixture was continuously stirred for $30 \mathrm{~min}$ before casting into stainless steel molds in order to avoid the flocculation, and storing at room temperature for two days to yield dry sheets. The visually uniform semi-transparent sheets were obtained, irrespective of C-PAM content. Basis weight of the sheets was ca. $30 \mathrm{~g} / \mathrm{m}^{2}$.

Papers containing C-PAM were prepared from beaten (CSF $510 \mathrm{ml}$ ) hardwood bleached kraft pulp and the previously described C-PAM by an internal application method ${ }^{7)}$. Total C-PAM content retained in the papers was determined by nitrogen analysis ${ }^{7,8)}$.

\subsection{Measurement of fundamental properties of sheets and papers}

As fundamental mechanical properties, tensile strength and Young's modulus were measured under strict condition of in-plane loading, according to the previous report ${ }^{7)}$. Thickness for the determination of sheet density and Young's modulus was measured by the rubber platen method according to TAPPI test method T551. Measurement of optical reflectance of specimens and calculation of scattering coefficient were referred to the previous report ${ }^{7)}$.

\subsection{Measurement of dynamic mechanical properties of sheets and papers ${ }^{10)}$}

Dynamic mechanical analysis (DMA) was conducted under nitrogen gas flow with Dynamic
Mechanical Spectrometer (DMS 6100, Seiko Instruments, Tokyo, Japan). The measurement was performed using oscillation mode at frequency of $10 \mathrm{~Hz}$ and a heating rate of $3{ }^{\circ} \mathrm{C} / \mathrm{min}$ with the temperature ranging from 120 to $300{ }^{\circ} \mathrm{C}$ to determine the dynamic storage modulus $\left(E^{\prime}\right)$, loss modulus ( $\left.E^{\prime \prime}\right)$, and loss tangent $\left(\tan \delta=E^{\prime \prime} / E^{\prime}\right)$. In order to measure these under linear viscoelasticity, $10 \mu \mathrm{m}$ and $50 \mathrm{mN}$ were selected as the dynamic oscillation amplitude and static tension, respectively. The test specimens were in rectangular shape with dimensions of $30 \mathrm{~mm}$ in length of span and $10 \mathrm{~mm}$ in width. In this experiment, the temperature was retained at a range from 100 to $120{ }^{\circ} \mathrm{C}$ for about $10 \mathrm{~min}$ before starting a measurement. Hence, it was not necessary to consider an influence of water content on the mechanical properties.

Table 1 Fundamental properties of MFC/C-PAM sheets and papers containing C-PAM.

\begin{tabular}{ccccccc}
\hline Sample & $\begin{array}{c}\text { C-PAM } \\
\text { content } \\
\%\end{array}$ & $\begin{array}{c}\text { Density } \\
\mathrm{kg} / \mathrm{m}^{3}\end{array}$ & $\begin{array}{c}\text { Scattering } \\
\text { coefficient } \\
\mathrm{m}^{2} / \mathrm{kg}\end{array}$ & $\begin{array}{c}\text { Tensile } \\
\text { index } \\
\mathrm{N} \cdot \mathrm{m} / \mathrm{g}\end{array}$ & $\begin{array}{c}\text { Young's } \\
\text { modulus } \\
\mathrm{GPa}\end{array}$ & $\begin{array}{c}\text { Stretch } \\
\text { at break } \\
\%\end{array}$ \\
\hline \hline \multirow{6}{*}{ MFC } & 0.0 & 1187 & 31.3 & 101 & 9.9 & 5.9 \\
& 1.0 & 1296 & 23.2 & 118 & 10.7 & 5.8 \\
& 2.0 & 1270 & 20.1 & 115 & 10.9 & 4.5 \\
& 3.0 & 1229 & 19.7 & 116 & 11.6 & 4.6 \\
& 5.0 & 1217 & 13.3 & 97 & 11.3 & 4.5 \\
\hline \multirow{4}{*}{ Paper } & 0.0 & 671 & 36.6 & 55 & 4.0 & 4.1 \\
& 0.5 & 671 & 36.2 & 70 & 4.3 & 4.9 \\
& 0.9 & 682 & 35.5 & 84 & 4.4 & 5.7 \\
& 1.8 & 695 & 34.3 & 87 & 4.5 & 5.9 \\
\hline
\end{tabular}

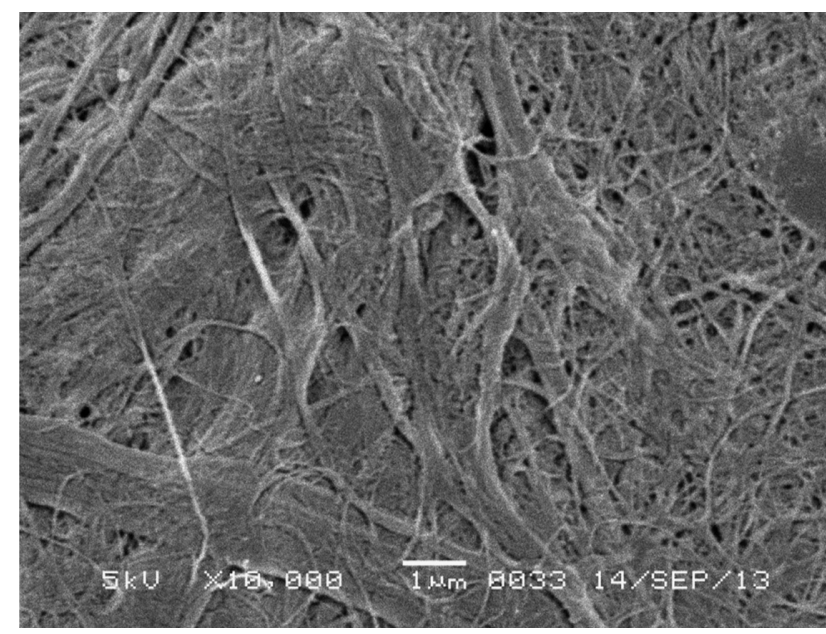

Fig. 1 SEM image of MFC/C-PAM sheet surface.

\section{Results and discussion}

\subsection{Fundamental properties}

Fundamental properties of the MFC/C-PAM sheets and papers containing C-PAM are given in Table 1 . Density of the MFC/C-PAM sheets was far higher than that of papers and was about twice, irrespective of CPAM content. Because the dimensions of MFC were essentially quite smaller (according to the information of 
the maker : $10-100 \mathrm{~nm}$ in width) than those of pulp fibers (ca. $10 \mu \mathrm{m}$ ), the sheets of MFC had higher consolidation than conventional papers, although the former included trunk fibrils with about $1 \mu \mathrm{m}$ width as shown in Fig.1. Scattering coefficient of the MFC/C-PAM sheets significantly decreased with an increase in C-PAM content. A similar trend of scattering power was reported for latex impregnated papers ${ }^{11)}$. Furthermore, a recent study showed that a higher C-PAM content in $\mathrm{MFC} / \mathrm{C}$ PAM sheets gave the high transparency ${ }^{5)}$. Generally, scattering coefficient means non-contact surface area surrounding the optically bonded fiber surface in paper, while modulus directly reflects the mechanically bonded fiber surface area which is surrounded by the optically bonded zone. Therefore, the significant decrease in scattering coefficient with increasing C-PAM content for the MFC/C-PAM sheets indicated an increase in the optically bonded area rather than a decrease in the noncontact area. That is to say, most C-PAM in the MFC/CPAM sheets could locate surrounding fibril-fibril bonds in a similar manner as rubber location surrounding fiberfiber bonds in latex impregnated papers ${ }^{11)}$.

Young's modulus of MFC sheet without C-PAM was significantly higher than that of papers, suggesting that the increase in mechanical bonded area between fibrils made by refining of fibrous materials was quite large. On the other hand, the increase in modulus with $\mathrm{C}$ PAM content is rather small for both MFC/C-PAM sheets and papers containing C-PAM, as was found in the previous study ${ }^{6}$. Thus, reinforcement of mechanical bonding with C-PAM for both could be caused by the increase in the modulus of the bonding, rather than an increase of mechanically bonded area.

Tensile strength of the MFC/C-PAM sheet without C-PAM was far larger than that of the paper, as expected from both moduli. However the strength increment of the MFC/C-PAM sheets with increasing C-PAM content was smaller than that expected from the difference of Young's modulus between the MFC/C-PAM sheets and the papers containing C-PAM. That is to say, the strength of MFC/ C-PAM sheets slightly increased with a further increase of C-PAM, and then rather decreased at the highest level of C-PAM content, while tensile strength of papers containing C-PAM significantly increased with a slight increase in C-PAM content. Taking into consideration that $\mathrm{Tg}$ of the C-PAM is ca $217^{\circ} \mathrm{C}$ (see Fig. 2) and the lower stretch at break of MFC/C-PAM sheets (see Table 1), these results may be derived from brittleness of C-PAM and MFC sheet themselves, thus stress concentration could easily occur in them and the film of
C-PAM was particularly fragile. A change in the shape of test specimen for tensile testing from rectangular to dumbbell shape may be needed for the MFC/C-PAM sheets with higher C- PAM content.

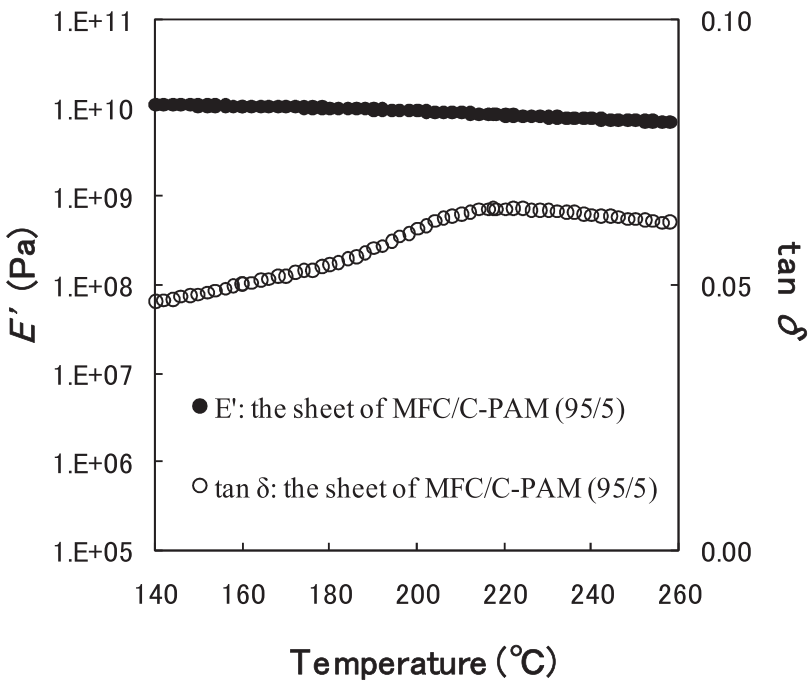

Fig. 2 Temperature dependence of $E^{\prime}(\bigcirc)$ and $\tan \delta$ (O) for the MFC/C-PAM sheet: MFC/C-PAM $=95 / 5$ on mass.

\subsection{Dynamic mechanical properties}

A typical example of the temperature dependence of $E^{\prime}$ and $\tan \delta$ for the MFC /C-PAM sheet (MFC/C-PAM = 95/5 on mass) is shown in Fig.2. The result was, on the whole, similar to that for the paper containing C-PAM ${ }^{10)}$, and thus suggested that the role of C-PAM on the mechanical bonding was unexpectedly small and the most of it was derived from fibril-fibril (hydrogen) bonding even in the MFC/C-PAM sheets. The temperature of the $\tan \delta$ peak of the MFC/C-PAM sheet was ca. $217{ }^{\circ} \mathrm{C}$, which agreed with that of a cast film of the corresponding C-PAM. In general, some changes in viscoelastic behavior, such as a shift of $\tan \delta$ peak temperature from that of the original polymer film, were usually detected as a sign of fiber-polymer interaction for paper-polymer composites $^{12)}$. The result in this study suggested that a certain interaction between MFC and C-PAM did not exist.

Because characteristic viscoelasticity most clearly appeared in $\tan \delta$ peak, the peak height was calculated by subtracting the value of $\tan \delta$ at $160{ }^{\circ} \mathrm{C}$ from the maximum value of $\tan \delta$ around $210{ }^{\circ} \mathrm{C}$ for each $\tan \delta$ curve as an index of viscoelastic behavior ${ }^{11}$. Relations between the peak height of $\tan \delta$ and C- PAM content for the $\mathrm{MFC} / \mathrm{C}-\mathrm{PAM}$ sheets and papers containing $\mathrm{C}-\mathrm{AM}$ are shown in Fig. 3. In this figure, general trends of the MFC/ C-PAM sheets agreed with those of the papers containing C-PAM, suggesting that viscoelastic behavior of the 


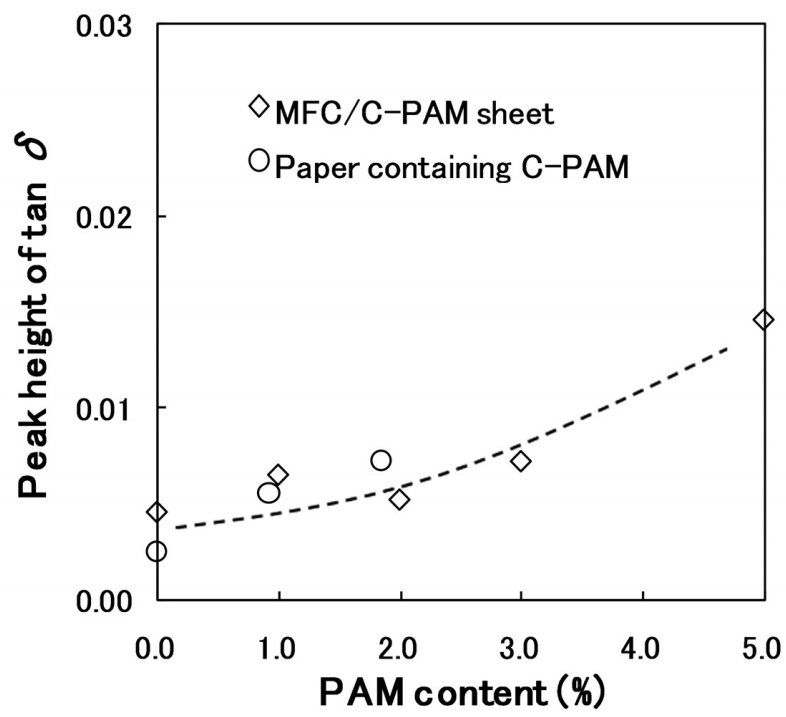

Fig. 3 Relations between the peak height of $\tan \delta$ and C-PAM content for the MFC/C-PAM sheets and papers containing C-PAM.

MFC/C-PAM sheets should reflect fiber-to-fiber bonds in papers containing C-PAM. That is to say, measurement of (dynamic) mechanical properties of casted sheets consisted of MFC and various water soluble polymers would be valuable to evaluate effects of the polymers on mechanical properties of papers when the polymers were used as dry strength agents.

\section{References}

1. M. A. Hubbe, O. J. Rojas, L. A. Lucia, and M. Sain, BioResources, 3, 929 (2008).

2. D. Sandquist, Appita J., 66, 156 (2013).

3. A. F. Turbak, F. Snyder, and K. Sandberg, J. Applied Polymer Sci. Applied Polymer Symp., 37, 815 (1983).

4. A. Isogai, SeniGakkaishi, 69, P-132 (2013).

5. T. Kurihara and A. Isogai, Cellulose, 21, 291 (2014).

6. T. Yamauchi and T. Hatanaka, Appita J., 55, 240 (2002).

7. T. Sakaemura and T. Yamauchi, Japan TAPPI J., 64, 1303 (2010).

8. T. Sakaemura, Doctor's Thesis (Kyoto University) (2010).

9. P. Myllytie, L. Salmen, E. Haimi, and J. Laine, Cellulose, 17, 375 (2010).

10. I. Mihara, T. Sakaemura and T. Yamauchi, J. Nordic Pulp Paper Res. J., 23, 382 (2008).

11. T. Yamauchi and T. Uenaka, Appita J., 58, 455 (2005).

12. T. Yamauchi, K. Murakami, and R. Imamura, Japan TAPPI J., 33, 473 (1979). 